\title{
KNOTS WITH BRAID INDEX THREE HAVE PROPERTY-P
}

\author{
W. MENASCO* and X. ZHANG ${ }^{\dagger}$ ‡ \\ Department of Mathematics, SUNY at Buffalo, Buffalo, NY 14260-2900, USA \\ *menasco@math.buffalo.edu \\ †xinzhang@math.buffalo.edu
}

Received 3 September 2001

\section{ABSTRACT}

We prove that knots with braid index three in the 3-sphere satisfy the Property $P$ conjecture.

\section{Introduction}

Let $K$ be a knot in the 3 -sphere $S^{3}$ and $M=M_{K}$ the complement of an open regular neighborhood of $K$ in $S^{3}$. As usual, the set of slopes on the torus $\partial M$ (i.e. the set of isotopy classes of unoriented essential simple loops on $\partial M$ ) is parameterized by

$$
\{m / n ; m, n \in \mathbb{Z}, n>0,(m, n)=1\} \cup\{1 / 0\},
$$

so that $1 / 0$ is the meridian slope of $K$ and $0 / 1$ is the longitude slope of $K$. The manifold obtained by Dehn surgery on $S^{3}$ along the knot $K$ (equivalently, Dehn filling on $M$ along the torus $\partial M)$ with slope $m / n$, is denoted by $K(m / n)$ or $M(m / n)$. Of course $K(1 / 0)=S^{3}$, and thus the surgery with the slope $1 / 0$ is called the trivial surgery. The celebrated Property $P$ conjecture, introduced by Bing and Matin in 1971 [2], states that every nontrivial knot $K$ in $S^{3}$ has Property P, i.e. every nontrivial surgery on $S^{3}$ along $K$ produces a non-simply connected manifold. For convenience we say that a class of knots in $S^{3}$ have Property $\mathrm{P}$ if every nontrivial knot in this class has Property P. The following classes of knots were known to have Property P: torus knots [12], symmetric knots [5] (the part for strongly invertible knots was proved in [4]), satellite knots [8], arborescent knots [17], alternating knots [6], and small knots with no non-integral boundary slopes [7]. For a simple homological reason, to prove the conjecture for a knot $K$ one only needs to consider the surgeries of $K$ with slopes $1 / n, n \neq 0$. A remarkable progress on the conjecture was made in [5]; it was proved there that for a nontrivial knot, only one of $K(1)$ or $K(-1)$ could possibly be a simply connected manifold. Another remarkable result

${ }^{\ddagger}$ Partially supported by NSF grant DMS 9971561. 
was given in [11], which told us that if the Property $\mathrm{P}$ conjecture is false, then the Poincare conjecture is false. For some earlier progresses on the conjecture, see [13, Problem 1.15] for a summary. In this paper we prove

Theorem 1. Knots in $S^{3}$ with braid index three satisfy the Property $P$ conjecture.

The main tools we shall use are the Casson invariant and essential laminations. We refer to [1] for the definition and basic properties of the Casson invariant and [10] for the definition and basic properties of an essential lamination. In Sec. 2 we give an outline of the proof of Theorem 1. Actually the proof of Theorem 1 is reduced there to that of two propositions, Proposition 3 and Proposition 4. These two propositions will then be proven in Secs. 3 and 4 respectively.

\section{Proof of Theorem 1}

Recall that the 3 -braid group, $B_{3}$, has the following well known Artin presentation:

$$
B_{3}=<\sigma_{1}, \sigma_{2} \mid \sigma_{1} \sigma_{2} \sigma_{1}=\sigma_{2} \sigma_{1} \sigma_{2}>
$$

where $\sigma_{1}$ and $\sigma_{2}$ are elementary 3-braids as shown in Fig. 1.

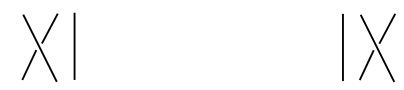

Fig. 1. $\sigma_{1}$ (the left figure) and $\sigma_{2}$ (the right figure).

If we let $a_{1}=\sigma_{1}, a_{2}=\sigma_{2}, a_{3}=\sigma_{1}^{-1} \sigma_{2} \sigma_{1}=\sigma_{2} \sigma_{1} \sigma_{2}^{-1}$, then $B_{3}$ also has the following presentation in generators $a_{1}, a_{2}$ and $a_{3}$ (see [18]):

$$
B_{3}=<a_{1}, a_{2}, a_{3} \mid a_{2} a_{1}=a_{3} a_{2}=a_{1} a_{3}>.
$$

In this paper we shall always express a 3 -braid as a word $w\left(a_{1}, a_{2}, a_{3}\right)$ in letters $a_{1}, a_{2}, a_{3}$. Such a word is called positive if the power of every letter in the word is positive. A positive word $w=a_{\tau_{1}} \cdots a_{\tau_{k}}$ is said to be in non-decreasing order $\left(N D\right.$-order) if the array of its subscripts $\left(\tau_{1}, \ldots, \tau_{k}\right)$ satisfies

$$
\tau_{j+1}=\tau_{j} \text { or } \tau_{j+1}=\tau_{j}+1\left(\bmod 3 \text { if } \tau_{j}+1=4\right) \text { for } j=1, \ldots, k-1 .
$$

One can define negative word and non-increasing order (NI-order) similarly. Let $P$ be the set of positive words in $N D$-order, let $N$ be the set of negative words in $N I$-order, and let $\alpha=a_{2} a_{1}$. It is proven in [18] that for any 3 -braid, there is a representative in its conjugacy class that is a shortest word in $a_{1}, a_{2}, a_{3}$ and is of the form

(i) a product of $\alpha^{k}$ and a word (maybe empty) in $P$ for some non-negative integer $k$; or 
(ii) a product of $\alpha^{k}$ and a word (maybe empty) in $N$ for some non-positive integer $k$; or

(iii) a product of a word in $P$ and a word in $N$,

where the meaning of the shortest is that the length, i.e. the number of letters of the representative is minimal among all representatives in the conjugacy class of the braid. Such representative of a 3-braid is said to be in normal form. We shall only need to show that if $K$ is a nontrivial knot in $S^{3}$ which is the closure of a 3-braid in normal form (i) or (ii) or (iii), then it has Property P.

Recall that a word $w\left(a_{1}, a_{2}, a_{3}\right)$ is called freely reduced if no adjacent letters are inverse to each other, and is called cyclically reduced if it is freely reduced and the first letter and the last letter of the word are not inverse to each other. Given a word $w\left(a_{1}, a_{2}, a_{3}\right)$, one can combine all adjacent letters of the same subscript into a single power of the letter, called a syllabus of the word in that subscript. A word $w$ is called syllabus reduced if it is expressed as a word in terms of syllabuses as

$$
w=a_{\tau_{1}}^{m_{1}} a_{\tau_{2}}^{m_{2}} \cdots a_{\tau_{k}}^{m_{k}}
$$

such that $a_{\tau_{j}} \neq a_{\tau_{j+1}}$ for $j=1, \ldots, k-1$. A word is called cyclically syllabus reduced if it is syllabus reduced and its first and last syllabuses are in different subscripts.

Let $P^{*}$ denote the set of all positive words in $a_{1}, a_{2}, a_{3}$ such that between any two syllabuses in $a_{3}$ both $a_{1}$ and $a_{2}$ occur. Obviously any 3-braid of norm form (i) is contained in $P^{*}$. Let $\beta$ be a 3 -braid in $P^{*}$. Suppose that $a_{3}^{k}$ is a syllabus in $\beta$ which is proceeded immediately by $a_{1}$. Then one can eliminate the syllabus $a_{3}^{k}$ with the equality $a_{1} a_{3}^{k}=a_{2}^{k} a_{1}$ to get an isotopic braid which is still in $P^{*}$ but with one less number of syllabuses in $a_{3}$. Similarly if a syllabus $a_{3}^{k}$ is followed immediately by $a_{2}$, then one can eliminate the syllabus $a_{3}^{k}$ with the equality $a_{3}^{k} a_{2}=a_{2} a_{1}^{k}$ to get an isotopic braid which is still in $P^{*}$ but with one less number of syllabuses in $a_{3}$. We shall call this process index-3 reduction. So for any given $\beta \in P^{*}$, we can find, after a finitely many times of index-3 reduction, an equivalent braid representative $\beta^{\prime}$ in $P^{*}$ for $\beta$ such that every syllabus in $a_{3}$ occurring in $\beta^{\prime}$ can only possibly be proceeded immediately by $a_{2}$ and likewise can only possibly be followed immediately by $a_{1}$. We call a word $\beta$ in $P^{*}$ index-3 reduced if every syllabus in $a_{3}$ occurring in $\beta$ is neither proceeded immediately by $a_{1}$ nor followed immediately by $a_{2}$.

Let $P^{a}$ denote the set of 3-braids of the form $\beta=a_{i}^{-q} \delta$, where $q=0$ or 1 and $\delta \in P^{*}$ is a non-empty word, such that $\delta$ is index-3 reduced and $\beta$ is cyclically syllabus reduced. Obviously $P$ is contained in $P^{a}$.

Proposition 2. Suppose that $K$ is a knot in $S^{3}$ which is the closure of a 3-braid $\beta=a_{i}^{-q} \delta$ in $P^{a}$ such that $\delta$ contains at least four syllabuses but $\beta$ is not one of the words in the set

$$
\begin{aligned}
E=\{ & a_{1}^{-1} a_{2} a_{3}^{2} a_{1} a_{2}, a_{1}^{-1} a_{3}^{2} a_{1} a_{2} a_{3}, a_{1}^{-1} a_{3} a_{1} a_{2}^{2} a_{3}, a_{1}^{-1} a_{2} a_{3} a_{1} a_{2}^{2}, \\
& a_{2}^{-1} a_{3} a_{1} a_{2} a_{3}^{2}, a_{2}^{-1} a_{3} a_{1}^{2} a_{2} a_{3}, a_{2}^{-1} a_{1} a_{2} a_{3}^{2} a_{1}, a_{2}^{-1} a_{1}^{2} a_{2} a_{3} a_{1}, \\
& \left.a_{3}^{-1} a_{1} a_{2} a_{3} a_{1}^{2}, a_{3}^{-1} a_{1} a_{2}^{2} a_{3} a_{1}, a_{3}^{-1} a_{2} a_{3} a_{1}^{2} a_{2}, a_{3}^{-1} a_{2}^{2} a_{3} a_{1} a_{2}\right\} .
\end{aligned}
$$


Then $K$ has positive Casson invariant and thus has Property $P$.

Later on we shall refer the set $E$ given in Proposition 2 as the excluded set.

Proposition 3. Suppose that $K$ is a knot in $S^{3}$ which is the closure of a 3-braid $\beta=\delta \eta$ which is in normal form (iii), i.e. $\delta \in P$ and $\eta \in N$. Suppose that either

1(1) each of $\delta$ and $\eta$ has a syllabus of power larger than one, or

(2) each of $\delta$ and $\eta$ contains at least two syllabuses, or

(3) one of $\delta$ and $\eta$ contains at least four syllabuses and the other has length at least two.

Then each of $K(1)$ and $K(-1)$ is a manifold which contains an essential lamination.

If a closed 3-manifold has an essential lamination, then its universal cover is $\mathbb{R}^{3}$ [10] and thus in particular the manifold cannot be simply connected. Hence any knot as given in Proposition 3 has Property $\mathrm{P}$ by [5].

Given Propositions 2 and 3, we can finish the proof of Theorem 1 as follows. For a braid $\beta$, we use $\hat{\beta}$ to denote the closure of $\beta$. Let $K \subset S^{3}$ be a nontrivial knot with index 3. Let $\beta$ be a 3-braid in normal form such that $\hat{\beta}=K$.
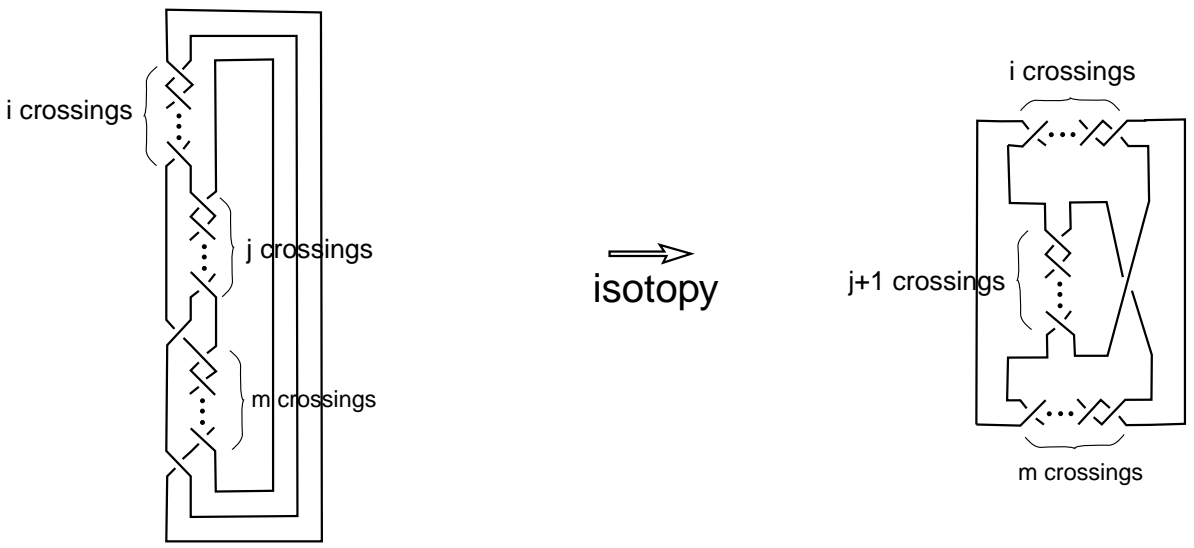

Fig. 2. The closure of $a_{1}^{i} a_{2}^{j} a_{3}^{m}$ is a Montesinos link.

First we consider the case that $\beta$ is in normal form (i), i.e. $\beta=\alpha^{k} \delta$ with $\delta \in P$ and $k \geq 0$. If $\beta$ contains at least four syllabuses and belongs to $P^{a}$, then $K=\hat{\beta}$ has positive Casson invariant by Proposition 2. So the knot $K$ has Property $\mathrm{P}$ in this case. If $\beta$ has less than four syllabuses, then up to conjugation in $B_{3}, \beta=a_{2} a_{1} a_{3}^{i}$ or $\beta=a_{1}^{i} a_{2}^{j} a_{3}^{m}$ for $i, j, m \geq 0$. It is easy to see that in this case $\hat{\beta}$ is an arborescent knot and thus by [17], $K=\hat{\beta}$ has Property P. For instance if $\beta=a_{1}^{i} a_{2}^{j} a_{3}^{m}$, then $\hat{\beta}$ is as shown in Fig. 2 which shows in fact that $\hat{\beta}$ is a Montesinos knot. So we may assume 
that $\beta$ has at least four syllabuses but is not in $P^{a}$. This implies that in $\beta=\alpha^{k} \delta$, we have $k>0$ and $\delta$ starts with a syllabus in $a_{3}$. Performing index-3 reduction on $\beta$ once, we get an equivalent 3 -braid $\beta^{\prime} \in P^{*}$ which is index-3 reduced, i.e. $\beta^{\prime} \in P^{a}$. If $\beta^{\prime}$ has less than four syllabuses, then again $K=\hat{\beta}=\hat{\beta}^{\prime}$ is an arborescent knot and thus has Property P. If $\beta^{\prime}$ contains at least four syllabuses, we may apply Proposition 2 to get Property $\mathrm{P}$ for the knot.

If $\beta$ is of normal form (ii), then the mirror image of $\beta$ is a braid of normal form (i) and thus the knot $K=\hat{\beta}$ has Property P.
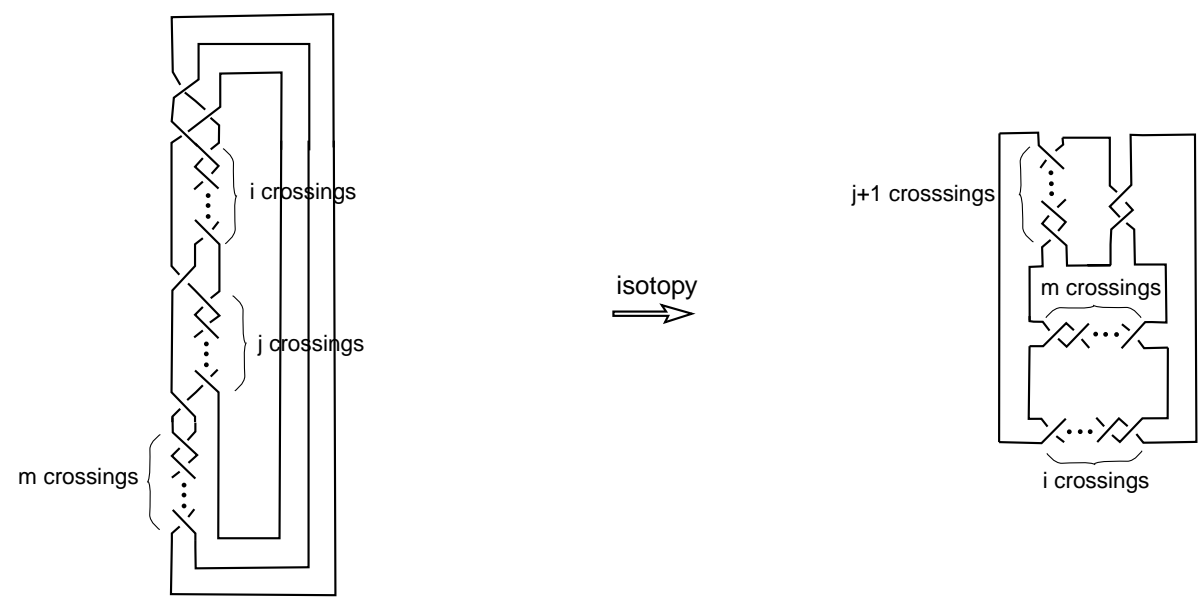

Fig. 3. The closure of $a_{3}^{-1} a_{2}^{i} a_{3}^{j} a_{1}^{m}$ is an arborescent link.

Suppose finally that $\beta=\delta \eta$ is a braid of normal form (iii) (recall that $\delta \in P$ and $\eta \in N)$. By Proposition 3, we may assume that each of the conditions (1)-(3) in Proposition 3 does not hold for $\beta=\delta \eta$. Then $\beta$ is a word of the form $\delta a_{i}^{-1}$ or $a_{i} \eta$, or $\beta=\delta a_{i}^{-k}$ where $k>1$ and $\delta$ contains at most three syllabuses each having power 1 , or $\beta=a_{1}^{k} \eta$ where $k>1$ and $\eta$ contains at most three syllabuses each having power -1 . If $\beta$ contains at most four syllabuses, then $K=\hat{\beta}$ is an arborescent knot and thus has Property P. (Figure 3 shows this for the case that $\beta=a_{3}^{-1} a_{2}^{i} a_{3}^{j} a_{1}^{m}$. Other cases can be treated similarly). So we may only consider the cases when $\beta=\delta a_{i}^{-1}$ or $\beta=a_{i} \eta$, each containing at least five syllabuses. If $\beta=\delta a_{i}^{-1}$, then $\beta$ is conjugate to $\beta^{\prime}=a_{i}^{-1} \delta$ which is in $P^{a}$. Hence if $\beta^{\prime}$ does not belong to the excluded set

$$
\begin{aligned}
E=\{ & a_{1}^{-1} a_{2} a_{3}^{2} a_{1} a_{2}, a_{1}^{-1} a_{3}^{2} a_{1} a_{2} a_{3}, a_{1}^{-1} a_{3} a_{1} a_{2}^{2} a_{3}, a_{1}^{-1} a_{2} a_{3} a_{1} a_{2}^{2}, \\
& a_{2}^{-1} a_{3} a_{1} a_{2} a_{3}^{2}, a_{2}^{-1} a_{3} a_{1}^{2} a_{2} a_{3}, a_{2}^{-1} a_{1} a_{2} a_{3}^{2} a_{1}, a_{2}^{-1} a_{1}^{2} a_{2} a_{3} a_{1}, \\
& \left.a_{3}^{-1} a_{1} a_{2} a_{3} a_{1}^{2}, a_{3}^{-1} a_{1} a_{2}^{2} a_{3} a_{1}, a_{3}^{-1} a_{2} a_{3} a_{1}^{2} a_{2}, a_{3}^{-1} a_{2}^{2} a_{3} a_{1} a_{2}\right\},
\end{aligned}
$$

then $K=\hat{\beta}^{\prime}$ has positive Casson invariant by Proposition 2 and thus has Property P. If $\beta^{\prime}$ is in the excluded set $E$, then $K=\hat{\beta}^{\prime}$ is an arborescent knot and thus has 

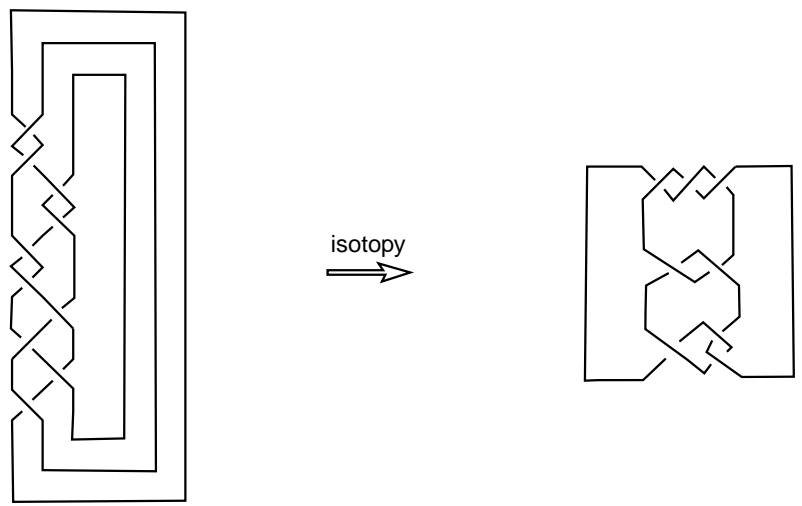

Fig. 4. The closure of the braid $a_{1}^{-1} a_{3}^{2} a_{1} a_{2} a_{3}$ is a Montesinos knot.

Property $\mathrm{P}$ [17]. (Figure 4 illustrates this for the case $\beta=a_{1}^{-1} a_{3}^{2} a_{1} a_{2} a_{3}$. Other cases can be checked similarly). Finally, if $\beta=a_{i} \eta$, then its mirror image is a braid in $P^{a}$, which is a case we have just discussed. This completes the proof of Theorem 1.

Propositions 2 and 3 will be proved in subsequent two sections which constitutes the rest of the paper.

\section{Proof of Proposition 2}

We retain all definitions and notations established earlier. For a 3 -braid $\beta$, we use $n_{\beta}$ to denote the number of syllabuses in $a_{3}$ occurring in $\beta$ and use $s_{\beta}$ to denote the number of syllabuses of $\beta$. Obviously if $\beta^{\prime}$ is the braid obtained from $\beta \in P^{*}$ after some non-trivial index-3 reduction, then $\hat{\beta}^{\prime}=\hat{\beta}$ but $n_{\beta^{\prime}}<n_{\beta}$. For a syllabus $a_{3}^{k}=a_{1}^{-1} a_{2}^{k} a_{1}$, we shall always assume its plane projection corresponds naturally to $a_{1}^{-1} a_{2}^{k} a_{1}$. Hence, every 3 -braid in letters $a_{1}, a_{2}, a_{3}$ has its canonical plane projection; namely in the projection plane we place vertically (from top to bottom) and successively the projections of letters occurring in the braid, corresponding to their natural order from left to right. Whenever we need consider a plane projection of a 3-braid, the canonical one is always assumed unless specifically indicated otherwise.
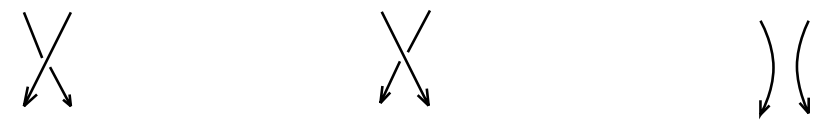

Fig. 5. $K_{+}$(the left figure), $K_{-}$(the middle figure) and $L_{0}$ (the right figure).

The basic tool we are going to use to prove the proposition is the following crossing change formula $(*)$ of the Casson invariant. If $K_{+}, K_{-}$are oriented knots and $L_{0}$ an oriented link with two components in $S^{3}$ such that they have identical plane projection except at one crossing they differ as shown in Fig. 5, then the 
Casson invariants $C_{K_{+}}$and $C_{K_{-}}$of $K_{+}$and $K_{-}$satisfy the following relation:

$$
C_{K_{+}}-C_{K_{-}}=l k\left(L_{0}\right),
$$

where $l k\left(L_{0}\right)$ is the linking number of $L_{0}$. This formula can be found on page 141 of [1] (note that there was a print error there, $K_{+}$and $K_{-}$in Figs. 36 and 37 of [1] should be exchanged). The idea of proof of the proposition is repeatedly applying the formula $(*)$ to a 3 -braid of the type as given in the proposition to reduce the complexity of the braid and inductively prove the positivity of its Casson invariant. To do so, we first need to estimate the linking number of a two-component link which is the closure of a 3-braid of relevant type. Given an oriented link $L$ of two components $L_{1}$ and $L_{2}$, we shall calculate the linking number of $L$ as follows. Take a plane projection of $L$. A crossing of $L$ as shown on the left of Fig. 5 has positive sign 1 and the crossing in the middle of the figure has negative sign -1 . Let $p$ be the number of positive crossings between of $L_{1}$ and $L_{2}$ and $q$ the number of negative crossings between $L_{1}$ and $L_{2}$. The linking number of $L$ is equal to $(p-q) / 2$. In other words, each positive (respectively negative) crossing between $L_{1}$ and $L_{2}$ contributes $1 / 2$ (respectively $-1 / 2$ ) to the linking number of $L$, and any crossing between $L_{1}$ and $L_{1}$ or between $L_{2}$ and $L_{2}$ contributes 0 to the linking number of $L$.

For a 3 -braid $\beta$, we shall always orient each component of $\hat{\beta}$ in such a way that the induced orientation on each strand of $\beta$ in its canonical projection is pointing downward in the projection plane. Suppose that $\hat{\beta}$ is a link of two components. If $\beta^{\prime}$ is a portion of $\beta$, we use $l\left(\beta^{\prime}\right)$ to denote the total contribution to the linking number of $\hat{\beta}$ coming from all the crossings of $\beta^{\prime}$, which maybe a half integer. In particular $l(\beta)=l k(\hat{\beta})$. Also if we decompose $\beta$ into portions $\beta=\beta_{1} \beta_{2} \cdots \beta_{k}$, then $l k(\hat{\beta})=l\left(\beta_{1}\right)+l\left(\beta_{2}\right)+\cdots+l\left(\beta_{k}\right)$.

Given the canonical plane projection of a 3 -braid $\eta$, we shall always call the strand of $\eta$ which starts at the top left corner the strand 1 of $\eta$, call the strand which starts at the top middle place the strand 2 of $\eta$, and call the strand which starts at the top right corner the strand 3 of $\eta$.

Lemma 4. Let $L=L_{1} \cup L_{2}$ be a link of two components in $S^{3}$ which is the closure of a braid $\beta=a_{i}^{-q} \delta$, where $q=0$ or 1 , and $\delta$ is a positive word in $a_{1}, a_{2}, a_{3}$. Then the linking number $l k(L)$ of $L$ is non-positive.

Proof. Note that $l\left(a_{i}\right) \leq 0$ for $i=1,2,3$, and $l\left(a_{i}^{-1}\right) \leq 1 / 2$ for $i=1,2$. Hence if in $\beta=a_{i}^{-q} \delta, q=0$ or $q=1$ but $i=1$ or 2 , then $l k(L)=l(\beta)=l\left(a_{i}^{-q}\right)+l(\delta) \leq 1 / 2$. But $l k(L)$ is an integer, so $l k(L) \leq 0$. So we may assume that $\beta=a_{3}^{-1} \delta$ and also assume that $l\left(a_{3}^{-1}\right)=1$. So in turn we may assume that the strand 1 of $a_{3}^{-1}$ belongs to $L_{1}$ and both strand 2 and 3 of $a_{3}^{-1}$ belong to $L_{2}$. One can then easily check that for any positive word $\delta$ in $a_{1}, a_{2}, a_{3}$, that one must have $l(\delta)<0$, in order to have a consistent link component assignment to the strands of $\beta$ (one can see this by just looking at the first one or two possible syllabuses of $\delta$ ). Hence $l k(L)=l(\beta) \geq 0$. 
We now prove Proposition 2. Let $\beta=a_{i}^{-q} \delta$ be a 3 -braid in $P^{a}$ as given in the proposition, whose closure is the given knot $K$. Recall that $s_{\delta} \geq 4$. Let $l_{\delta}$ denote the total length of $\delta$. The stratege of the proof is to use induction on the total length $l_{\delta}$ of a knot from the set all braids $\beta=a^{-1} \delta$ in $P^{a}$ with $s_{\delta} \geq 4$, applying the crossing change formula for Casson invariant.

By hand calculation case by case, one can check that Proposition 2 holds for all braids $\beta=a_{i}^{-q} \delta \in P^{a}$ with $s_{\delta} \geq 4$ and $l_{\delta} \geq 5$. So we may assume that a given $\beta=a_{i}^{-1} \delta \in P^{a}$ has $s_{\delta} \geq 4$ and $l_{\delta} \geq 6$.

If some syllabus in $a_{3}$ occurring in $\delta$ has power $k>2$, we apply the crossing change formula $(*)$ for Casson invariant to $K=\hat{\beta}$ at the second crossing of the syllabus. The link $L$ (of two components) obtained by smoothing the crossing is the closure of a braid of the type as described in Lemma 4 and thus has non-positive linking number. So we get a new braid $\beta^{\prime}=a_{i}^{-q} \delta^{\prime}$ which is identical with $\beta$ except with two less in power at the syllabus in $a_{3}$ and the Casson invariant of $\hat{\beta}^{\prime}$ is less than or equal to that of $\hat{\beta}$. Obviously $\beta^{\prime}$ is still in $P^{a}$. So if $l_{\delta} \geq 6$, we may apply induction. If $l_{\delta^{\prime}}<6$ we may check directly that the original braid $\hat{b}$ has positive Casson invariant. So we may assume that every syllabus in $a_{3}$ has power at most two. Similarly we may assume that the power of a syllabus in $a_{2}$ or in $a_{1}$ is one or two.

Claim D1. If $a_{3}^{2}$ is a syllabus of $\delta$, then the Proposition holds.

Proof. Consider the first such syllabus occurring in $\delta$. Applying the formula $(*)$ to $\hat{\beta}$ at the second crossing of the syllabus $a_{3}^{2}$, we get a new braid $\beta^{\prime}=a_{i}^{-q} \delta^{\prime}$, where $\delta^{\prime}$ is the braid obtained from $\delta$ by deleting the syllabus $a_{3}^{2}$, such that $\delta^{\prime} \in P^{*}$, $n_{\delta^{\prime}}=n_{\delta}-1$, and $C_{\hat{\beta}} \geq C_{\hat{\beta}^{\prime}}$ (by Lemma 4). If $\beta^{\prime}$ is still in $P^{a}$ and $\delta^{\prime}$ contains at least four syllabuses but $\beta^{\prime}$ is not a word in the excluded set $E$, then we may use induction.

Suppose that $\beta^{\prime} \in E$. Since the syllabus we are considering is the first such occurring in $\beta$ and since $\delta$ is index- 3 reduced, $\beta$ can only be the word $a_{2}^{-1} a_{3}^{2} a_{1} a_{2} a_{3}^{2} a_{1}$ or $a_{2}^{-1} a_{3}^{2} a_{1}^{2} a_{2} a_{3} a_{1}$ or $a_{2}^{-1} a_{3}^{2} a_{1} a_{2} a_{3} a_{1}^{2}$ or $a_{1}^{-1} a_{2} a_{3} a_{1} a_{2}^{2} a_{3}^{2}$. In such a case one can verify directly that $C_{\hat{\beta}}$ is positive.

Hence, we may assume that either $\beta^{\prime}$ is still in $P^{a}$ with $\delta^{\prime}$ containing exactly three syllabuses, or $\beta^{\prime}$ is no longer in $P^{a}$. In the former case, $\beta$ is a word in the set

$$
\left\{\begin{array}{lllll}
a_{1}^{j} a_{2}^{k} a_{3}^{2} a_{1}^{m}, & a_{2}^{j} a_{1}^{k} a_{2}^{m} a_{3}^{2}, & a_{2}^{j} a_{3}^{2} a_{1}^{k} a_{2}^{m}, & a_{3}^{2} a_{1}^{k} a_{2}^{j} a_{3}^{m}, & a_{3}^{2} a_{1}^{k} a_{2}^{j} a_{1}^{m}, \\
a_{3} a_{1}^{k} a_{2}^{m} a_{3}^{2}, & a_{1}^{-1} a_{2}^{j} a_{1}^{k} a_{2}^{m} a_{3}^{2}, & a_{1}^{-1} a_{2}^{j} a_{3}^{2} a_{1}^{k} a_{2}^{m}, & a_{1}^{-1} a_{3} a_{1}^{k} a_{2}^{m} a_{3}^{2}, & a_{2}^{-1} a_{1}^{j} a_{2}^{k} a_{3}^{2} a_{1}^{m}, \\
a_{2}^{-1} a_{3}^{2} a_{1}^{j} a_{2}^{k} a_{3}^{m}, & a_{2}^{-1} a_{3}^{2} a_{1}^{k} a_{2}^{j} a_{1}^{m}, & a_{3}^{-1} a_{1}^{j} a_{2}^{k} a_{3}^{2} a_{1}^{m}, & a_{3}^{-1} a_{2}^{j} a_{3}^{2} a_{1}^{k} a_{2}^{m} &
\end{array}\right\}
$$

for some $j, k, m \in\{1,2\}$. When $\beta$ is one of words in this set but is not in the excluded set $E$, one can verify directly using the formula $(*)$ that $\hat{\beta}$ has positive Casson invariant. For instance, when $\beta=a_{1}^{-1} a_{2}^{j} a_{1}^{k} a_{2}^{m} a_{3}^{2}$, it is conjugate to $a_{2}^{j} a_{1}^{k} a_{2}^{m} a_{3}^{2} a_{1}^{-1}=$ $a_{2}^{j} a_{1}^{k} a_{2}^{m} a_{1}^{-1} a_{2}^{2}$ which in turn is conjugate to $a_{1}^{-1} a_{2}^{j+2} a_{1}^{k} a_{2}^{m}$. So we need to show that the Casson invariant of the closure of the braid $\eta=a_{1}^{-1} a_{2}^{j+2} a_{1}^{k} a_{2}^{m}$ is positive. We 
have eight possible cases for $\eta$ corresponding to various possible values of $j, k$ and $m$. But only in case $j=k=m=1$ or case $j=1, k=m=2$ or case $j=k=2, m=1$, the closure of the involved braid is a knot, and in such a case one can verify directly using formula $(*)$ that $\hat{\beta}=\hat{\eta}$ has positive Casson invariant. In a similar way one can verify the proposition for each of the other words in the above set. (We did the checking!)

We now consider the latter case when $\beta^{\prime}$ is not in $P^{a}$. It follows that the syllabus $a_{3}^{2}$ is either the first or the last syllabus of $\delta$ and $q=1$. We consider the case when the syllabus $a_{3}^{2}$ is the first syllabus of $\delta$. The case when the syllabus $a_{3}^{2}$ is the last syllabus of $\delta$ can be treated similarly. It follows that $\beta=a_{1}^{-1} a_{3}^{2} a_{1}^{j} a_{2}^{k} \cdots$, for some $j, k \in\{1,2\}$, and $\beta$ does not end with $a_{1}$. If $j=2$, then $\beta^{\prime}=a_{1}^{-1} a_{1}^{2} a_{2}^{k} \cdots$ which is isotopic to $\beta^{\prime \prime}=a_{1} a_{2}^{k} \cdots$ which is in $P^{a}$. So if $\beta^{\prime \prime}$ contains at least four syllabuses, we may use the induction or check $C_{\hat{\beta}}$ directly. We may then assume that $\beta^{\prime \prime}$ has less than four syllabuses. It follows that $\beta^{\prime \prime}=a_{1} a_{2}^{k} a_{3}^{m}$ which is a knot only when $k=2, m=1$ or $k=1, m=2$ and in these two cases $C_{\hat{\beta}} \geq C_{\hat{\beta}^{\prime \prime}}>0$. So we may assume that $j=1$. In this case $\beta^{\prime}$ is isotopic to $\beta^{\prime \prime}=a_{2}^{k} \cdots$ which is in $P^{a}$. Hence if $\beta^{\prime \prime}$ contains at least four syllabuses, we may use the induction. If $\beta^{\prime \prime}$ has less than four syllabuses, then we must either have $\beta=a_{1}^{-1} a_{3}^{2} a_{1} a_{2}^{k} a_{1}^{n} a_{2}^{m}$ or $\beta=a_{1}^{-1} a_{3}^{2} a_{1} a_{2}^{m} a_{3}^{j}$. In the former case, $\beta^{\prime \prime}$ has positive Casson invariant (a nontrivial positive knot). In the latter case, only when $m=1, j=1, \hat{\beta}$ is a knot. But this braid is in the set $E$. The proof of Claim D1 is now complete.

By Claim D1, we may now assume that every syllabus in $a_{3}$ occurring in $\delta$ has power equal to one.

Claim D2. We may assume that every syllabus in $a_{1}$ occurring in $\delta$ has power equal to one.

Proof. Suppose that $\delta$ contains syllabuses in $a_{1}$ of power two. Consider the first such syllabus occurring in $\delta$. Applying the formula $(*)$ to $\hat{\beta}$ at the first crossing of the syllabus $a_{1}^{2}$, we get a new braid $\beta^{\prime}=a_{i}^{-q} \delta^{\prime}$ such that the Casson invariant of $\hat{\beta}^{\prime}$ is less than or equal to that of $\hat{\beta}$ by Lemma 4 . Obviously $\delta^{\prime}$ is still a positive word in $a_{1}, a_{2}, a_{3}$ but may not be in $P^{*}$ or $P^{a}$. We have several possibilities for $\delta$ around the given syllabus $a_{1}^{2}: \delta=\cdots a_{2}^{j} a_{1}^{2} a_{2}^{k} \cdots$ or $\delta=\cdots a_{3} a_{1}^{2} a_{2}^{k} \cdots$ or $\delta=a_{1}^{2} a_{2}^{k} \cdots$ or $\delta=\cdots a_{1}^{2}$, for some $j, k \in\{1,2\}$.

Case (D2.1). $\delta=\cdots a_{2}^{j} a_{1}^{2} a_{2}^{k} \cdots$.

Then $\delta^{\prime}=\cdots a_{2}^{j+k} \cdots$ and $\beta^{\prime}=a_{i}^{-q} \delta^{\prime}$ is still in $P^{a}$. Also if $j+k>2$, we may apply the formula $(*)$ one more time to bring it down to one or two. Let $\beta^{\prime \prime}=a_{i}^{-q} \delta^{\prime \prime}$ be the resulting braid. Then if $\delta^{\prime \prime}$ contains at least four syllabuses, we may use the induction or check $C_{\hat{\beta}}$ directly. If $\beta^{\prime \prime}$ is in $E$, then one can verify directly that the original braid $\beta$ has positive Casson invariant. Note that $s_{\delta^{\prime}}=s_{\delta}-2$. Suppose that $s_{\delta^{\prime}}<4$. Then $s_{\delta}$ is four or five and $\beta=a_{i}^{-q} a_{2}^{j} a_{1}^{2} a_{2}^{k} a_{3}$ or $\beta=a_{i}^{-q} a_{1} a_{2}^{j} a_{1}^{2} a_{2}^{k} a_{3}$ or 
$\beta=a_{i}^{-q} a_{3} a_{1} a_{2}^{j} a_{1}^{2} a_{2}^{k}$. Easy to check that when $q=0$, any knot from these cases has positive Casson invariant. So assume that $q=1$ in these cases.

If $\beta=a_{i}^{-1} a_{2}^{j} a_{1}^{2} a_{2}^{k} a_{3}$, then $i=1$ since $\beta$ is cyclically reduced. So $\beta=$ $a_{1}^{-1} a_{2}^{j} a_{1}^{2} a_{2}^{k} a_{3}$. To be a knot, we have $k=j=1$ or $k=j=2$. In each of the two cases, one can verify directly that the Casson invariant of the knot is positive.

If $\beta=a_{i}^{-q} a_{1} a_{2}^{j} a_{1}^{2} a_{2}^{k} a_{3}$, then $i=2$. That is $\beta=a_{2}^{-1} a_{1} a_{2}^{j} a_{1}^{2} a_{2}^{k} a_{3}$. One can also directly verify that $C_{\hat{\beta}}>0$ (for those values of $k, j \in\{1,2\}$ which make $\hat{\beta}$ a knot).

The case that $\beta=a_{i}^{-q} a_{3} a_{1} a_{2}^{j} a_{1}^{2} a_{2}^{k}$ can be treated similarly.

Case (D2.2). $\delta=\cdots a_{3} a_{1}^{2} a_{2}^{k} \cdots$.

Then $\delta^{\prime}=\cdots a_{3} a_{2}^{k} \cdots=\cdots a_{2} a_{1} a_{2}^{k-1} \cdots$.

Case (D2.2.1.) $k=2$.

If $\delta$ does not start with $a_{3}$, then $\beta^{\prime}=a_{i}^{-q} \delta^{\prime}=a_{i}^{-q} \cdots a_{2}^{j+1} a_{1} a_{2} \cdots$ is still in $P^{a}$ and is not in the set $E$. In such case if $\delta^{\prime}$ contains at least four syllabuses, we may apply induction or check $C_{\hat{\beta}}$ directly. If $s_{\delta^{\prime}}<4$, then $\beta=a_{i}^{-q} a_{2}^{j} a_{3} a_{1}^{2} a_{2}^{2}$. To be a knot, we have $q=0, j=1$ or $q=1, i=1, j=2$. In each of the two cases we have $C_{\hat{\beta}}>0$ by direct calculation.

If $\delta$ starts with $a_{3}$ but $q=0$, then $\beta^{\prime}=\delta^{\prime}$ is still in $P^{a}$ but not in $E$. Also $s_{\beta}=s_{\delta}=s_{\beta^{\prime}}=s_{\delta^{\prime}}$. So we may apply induction or check $C_{\hat{\beta}}$ directly.

If $\delta$ starts with $a_{3}$ and $q=1$, then $i=1$ or 2 . If $i=1$, then $\beta^{\prime}=a_{1}^{-1} a_{2} a_{1} a_{2} \ldots$ is still in $P^{a}$ but not in $E$ and contains at least five syllabuses. So we may use induction. If $i=2$, then $\beta^{\prime}=a_{1} a_{2} \cdots$ is in $P^{a}$ and is not in $E$. Also $s_{\beta^{\prime}}=s_{\delta}-$ 1. Hence if $s_{\delta}>4$, we may use induction. So suppose that $s_{\delta}=4$. Then $\beta=$ $a_{2}^{-1} a_{3} a_{1}^{2} a_{2}^{2} a_{3}$ or $\beta=a_{2}^{-1} a_{3} a_{1}^{2} a_{2}^{2} a_{1}^{j}$. But the former is not a knot. The latter is a knot when $j=2$, in which case $C_{\hat{\beta}}>0$.

Case (D2.2.2). $k=1$.

Then $\delta=\cdots a_{3} a_{1}^{2} a_{2} a_{3} \cdots$ or $\delta=\cdots a_{3} a_{1}^{2} a_{2} a_{1}^{j} \cdots$ or $\delta=\cdots a_{3} a_{1}^{2} a_{2}$. Correspondingly, we have $\delta^{\prime}=\cdots a_{2} a_{1} a_{3} \cdots=\cdots a_{2}^{2} a_{1} \cdots$ or $\delta=\cdots a_{2} a_{1}^{j+1} \cdots$ or $\delta=\cdots a_{2} a_{1}$. If $\beta^{\prime}$ is in $P^{a}-E$ and $s_{\delta^{\prime}} \geq 4$, we may use induction. If $\beta^{\prime} \in E$, then one can check that the old braid $\beta$ has positive Casson invariant. If $\beta^{\prime} \in P^{a}$ but $s_{\delta^{\prime}}<4$, then one can also verify that $\beta$ always has positive Casson invariant, applying the conditions that (1) $\beta \in P^{a}-E,(2) s_{\delta} \geq 4$ and (3) $\hat{\beta}$ is a knot.

Case (D2.3). $\delta=a_{1}^{2} a_{2}^{k} \cdots$.

Then $\delta=a_{1}^{2} a_{2}^{k} a_{1}^{j} a_{2}^{m} \cdots$ or $\delta=a_{1}^{2} a_{2}^{k} a_{3} a_{1}^{j} \ldots$. And $\delta^{\prime}=a_{2}^{k} a_{1}^{j} a_{2}^{m} \ldots$ or $\delta^{\prime}=$ $a_{2}^{k} a_{3} a_{1}^{j} \ldots$. So $\beta^{\prime}$ is in $P^{a}$ unless $\beta$ starts with $a_{2}^{-1}$. If $\beta$ starts with $a_{2}^{-1}$, then $\beta^{\prime}=a_{2}^{k-1} a_{1}^{j} a_{2}^{m} \cdots$ or $\beta^{\prime}=a_{2}^{k-1} a_{3} a_{1}^{j} \cdots$ which is in $P^{a}$. Again in each of these cases, if $\beta^{\prime}$ is the set $E$ or $s_{\delta^{\prime}}<4$, one can calculate directly that the old braid $\beta$ has positive Casson invariant. Otherwise one can use the induction.

Case (D2.4). $\delta=\cdots a_{1}^{2}$.

This case can be treated similarly as in the previous case. 
So by Claims D1 and D2, we now assume that every syllabus in $a_{3}$ and in $a_{1}$ occurring in $\delta$ have power equal to one.

Claim D3. We may assume that every syllabus in $a_{2}$ occurring in $\delta$ has power equal to one.

Proof. This can be proved similarly as Claim D2.

So we now assume that every syllabus occurring in $\delta$ has power one.

If $a_{1} a_{2} a_{1}$ appears immediately after an $a_{3}$, then $\beta=\cdots a_{3} a_{1} a_{2} a_{1} \cdots=$ $\cdots a_{3} a_{1} a_{3} a_{2} \cdots=\cdots a_{3} a_{2} a_{1} a_{2} \cdots=\cdots a_{2} a_{1}^{2} a_{2} \cdots$ which is still in $P^{a}$. Hence we may apply Claim D2 $\beta$ is in the set $\left\{a_{3} a_{1} a_{2} a_{1}, a_{2}^{-1} a_{3} a_{1} a_{2} a_{1}, a_{3}^{-1} a_{2} a_{3} a_{1} a_{2} a_{1}\right.$, $\left.a_{1}^{-1} a_{3} a_{1} a_{2} a_{1} a_{2}, a_{1}^{-1} a_{2} a_{3} a_{1} a_{2} a_{1} a_{2}, a_{3}^{-1} a_{2} a_{3} a_{1} a_{2} a_{1} a_{2}\right\}$. If $\beta$ is a word in this set, then either $\hat{\beta}$ is not a knot or $\hat{\beta}$ has positive Casson invariant. So we may assume that no $a_{3} a_{1} a_{2} a_{1}$ occurs in $\beta$. A similar argument shows that we may assume that no $a_{2} a_{1} a_{2} a_{3}$ occurs in $\beta$. Hence we may assume that $\beta$ is one of the words in the set

$\left\{\begin{array}{llll}a_{1} a_{2} a_{3} a_{1}, & \left(a_{3} a_{1} a_{2}\right)^{m}, & \left(a_{3} a_{1} a_{2}\right)^{m} a_{3}, & \left(a_{3} a_{1} a_{2}\right)^{m} a_{3} a_{1}, \\ a_{2}\left(a_{3} a_{1} a_{2}\right)^{m}, & a_{1} a_{2}\left(a_{3} a_{1} a_{2}\right)^{m}, & a_{2}\left(a_{3} a_{1} a_{2}\right)^{m} a_{3}, & a_{2}\left(a_{3} a_{1} a_{2}\right)^{m} a_{3} a_{1}, \\ a_{1} a_{2}\left(a_{3} a_{1} a_{2}\right)^{m} a_{3}, & a_{1} a_{2}\left(a_{3} a_{1} a_{2}\right)^{m} a_{3} a_{1}, & a_{2}^{-1} a_{1} a_{2} a_{3} a_{1}, & a_{3}^{-1} a_{1} a_{2} a_{3} a_{1}, \\ a_{1}^{-1}\left(a_{3} a_{1} a_{2}\right)^{m}, & a_{1}^{-1}\left(a_{3} a_{1} a_{2}\right)^{m} a_{3}, & a_{2}^{-1}\left(a_{3} a_{1} a_{2}\right)^{m} a_{3}, & a_{2}^{-1}\left(a_{3} a_{1} a_{2}\right)^{m} a_{3} a_{1} \\ a_{1}^{-1} a_{2}\left(a_{3} a_{1} a_{2}\right)^{m}, & a_{3}^{-1} a_{2}\left(a_{3} a_{1} a_{2}\right)^{m}, & a_{3}^{-1} a_{1} a_{2}\left(a_{3} a_{1} a_{2}\right)^{m}, & a_{1}^{-1} a_{2}\left(a_{3} a_{1} a_{2}\right)^{m} a_{3}, \\ a_{3}^{-1} a_{2}\left(a_{3} a_{1} a_{2}\right)^{m} a_{3} a_{1} & a_{2}^{-1} a_{1} a_{2}\left(a_{3} a_{1} a_{2}\right)^{m} a_{3}, a_{2}^{-1} a_{1} a_{2}\left(a_{3} a_{1} a_{2}\right)^{m} a_{3} a_{1}, & a_{3}^{-1} a_{1} a_{2}\left(a_{3} a_{1} a_{2}\right)^{m} a_{3} a_{1}\end{array}\right\}$

where $m>0$.

If $\beta=a_{1} a_{2} a_{3} a_{1}$, then it has positive Casson invariant. The case $\beta=\left(a_{3} a_{1} a_{2}\right)^{m}$ cannot occur since its closure is not a knot for all $m>0$. Similarly each of the cases

$$
\begin{gathered}
a_{2}\left(a_{3} a_{1} a_{2}\right)^{m} a_{3} a_{1}, a_{1} a_{2}\left(a_{3} a_{1} a_{2}\right)^{m} a_{3}, a_{2}^{-1} a_{1} a_{2} a_{3} a_{1}, \\
a_{3}^{-1} a_{1} a_{2} a_{3} a_{1}, a_{1}^{-1}\left(a_{3} a_{1} a_{2}\right)^{m}, a_{2}^{-1}\left(a_{3} a_{1} a_{2}\right)^{m} a_{3} a_{1} \text { and } a_{1}^{-1} a_{2}\left(a_{3} a_{1} a_{2}\right)^{m} a_{3}
\end{gathered}
$$

cannot occur as $\beta$. If $\beta=\left(a_{3} a_{1} a_{2}\right)^{m} a_{3}$, then it is conjugate to $\beta^{\prime}=$ $a_{3}^{2} a_{1} a_{2}\left(a_{3} a_{1} a_{2}\right)^{m-1}$. So we may apply Claim D1 unless $m=1$. But when $m=1$, one can calculate directly that $\beta=a_{3} a_{1} a_{2} a_{3}$ has positive Casson invariant. If $\beta=\left(a_{3} a_{1} a_{2}\right)^{m} a_{3} a_{1}$, then it is conjugate to $\beta^{\prime}=a_{2} a_{1}^{3} a_{2}\left(a_{3} a_{1} a_{2}\right)^{m-1}$. So we are back to a previous case unless $m=1$. But when $m=1, \hat{\beta}$ is not a knot. If $\beta=a_{2}\left(a_{3} a_{1} a_{2}\right)^{m}$, then it is conjugate to $\beta^{\prime}=\left(a_{3} a_{1} a_{2}\right)^{m-1} a_{3} a_{1} a_{2}^{2}$. So we may apply Claim D2 unless $m=1$. But when $m=1$, one can calculate directly that $\beta=a_{2} a_{3} a_{1} a_{2}$ has positive Casson invariant. Similarly one can deal with the cases $a_{1} a_{2}\left(a_{3} a_{1} a_{2}\right)^{m}, a_{2}\left(a_{3} a_{1} a_{2}\right)^{m} a_{3}$ and $a_{1} a_{2}\left(a_{3} a_{1} a_{2}\right)^{m} a_{3} a_{1}$.

Suppose that $\beta=a_{1}^{-1}\left(a_{3} a_{1} a_{2}\right)^{m} a_{3}$. To be a knot, we have $m \geq 3$ and $m=3$ $(\bmod 2)$. Also $\beta$ is conjugate to $\beta^{\prime}=a_{1}^{-1} a_{2}\left(a_{3} a_{1} a_{2}\right)^{m}$ which has less number of syllabuses in $a_{3}$ and thus we may apply the induction. Similarly one can deal with the case when $\beta=a_{2}^{-1}\left(a_{3} a_{1} a_{2}\right)^{m} a_{3}$.

If $\beta=a_{1}^{-1} a_{2}\left(a_{3} a_{1} a_{2}\right)^{m}$, then to be a knot $m$ must be even. Applying the formula $(*)$ at the first crossing of $\beta$, we get $C_{\hat{\beta}}=C_{\hat{\beta}_{1}}+l k\left(\hat{\lambda}_{1}\right)$ where $\beta_{1}=a_{1} a_{2}\left(a_{3} a_{1} a_{2}\right)^{m}$ 
and $\lambda_{1}=a_{2}\left(a_{3} a_{1} a_{2}\right)^{m}$. One can easily deduce from the projection of $\lambda_{1}$ that $l k\left(\hat{\lambda}_{1}\right)=-m / 2$. Hence, we have $C_{\hat{\beta}}=C_{\hat{\beta}_{1}}-m / 2$. In such case it suffices to show the following.

Claim D4. $C_{\hat{\beta}_{1}}>m / 2$.

Proof. We knew that $m=2 p$ with $p>0$. We shall prove the claim by induction on the number $p$. Write $\beta_{1}$ as $\beta_{1}=a_{1} a_{2} a_{1}^{-1} a_{2} a_{1}^{2} a_{2} a_{1}^{-1} a_{2} a_{1}^{2} a_{2}\left(a_{3} a_{1} a_{2}\right)^{m-2}$. Applying formula $(*)$ to $\beta_{1}$ at the first crossing of the first syllabus $a_{1}^{2}$, we get $C_{\hat{\beta}_{1}}=C_{\hat{\beta}_{2}}-l k\left(\hat{\lambda}_{2}\right)$ where $\beta_{2}=a_{1} a_{2} a_{1}^{-1} a_{2}^{2} a_{1}^{-1} a_{2} a_{1}^{2} a_{2}\left(a_{3} a_{1} a_{2}\right)^{m-2}$ and $\lambda_{2}=$ $a_{1} a_{2} a_{1}^{-1} a_{2} a_{1} a_{2} a_{1}^{-1} a_{2} a_{1}^{2} a_{2}\left(a_{3} a_{1} a_{2}\right)^{m-2}$. One can easily deduce from the projection of $\lambda_{2}$ that $l k\left(\hat{\lambda}_{2}\right)=-2(m-2)-3=-4 p+1$. Hence, we have $C_{\hat{\beta}_{1}}=C_{\hat{\beta}_{2}}+4 p-1$. We then apply formula $(*)$ to $\beta_{2}$ at the first crossing of the first syllabus $a_{2}^{2}$, we get $C_{\hat{\beta}_{2}}=C_{\hat{\beta}_{3}}-l k\left(\hat{\lambda}_{3}\right)$ where $\beta_{3}=a_{1} a_{2} a_{1}^{-2} a_{2} a_{1}^{2} a_{2}\left(a_{3} a_{1} a_{2}\right)^{m-2}$ and $\lambda_{3}=$ $a_{1} a_{2} a_{1}^{-1} a_{2} a_{1}^{-1} a_{2} a_{1}^{2} a_{2}\left(a_{3} a_{1} a_{2}\right)^{m-2}$. One can calculate to see that $l k\left(\hat{\lambda}_{3}\right)=-p-1$. Hence, we have $C_{\hat{\beta}_{2}}=C_{\hat{\beta}_{3}}+p+1$. We then apply formula $(*)$ to $\beta_{3}$ at the first crossing of the syllabus $a_{1}^{-2}$, we get $C_{\hat{\beta}_{3}}=C_{\hat{\beta}_{4}}+l k\left(\hat{\lambda}_{4}\right)$ where $\beta_{4}=a_{1} a_{2}^{2} a_{1}^{2} a_{2}\left(a_{3} a_{1} a_{2}\right)^{m-2}$ and $\lambda_{4}=a_{1} a_{2} a_{1}^{-1} a_{2} a_{1}^{2} a_{2}\left(a_{3} a_{1} a_{2}\right)^{m-2}$. Also one can calculate to see that $l k\left(\hat{\lambda}_{4}\right)=$ $-p$. Hence, we have $C_{\hat{\beta}_{3}}=C_{\hat{\beta}_{4}}-p$. We then apply formula $(*)$ to $\beta_{4}$ at the first crossing of the syllabus $a_{2}^{2}$, we get $C_{\hat{\beta}_{4}}=C_{\hat{\beta}_{5}}-l k\left(\hat{\lambda}_{5}\right)$ where $\beta_{5}=a_{1}^{3} a_{2}\left(a_{3} a_{1} a_{2}\right)^{m-2}$ and $\lambda_{5}=a_{1} a_{2} a_{1}^{2} a_{2}\left(a_{3} a_{1} a_{2}\right)^{m-2}$. We also have $l k\left(\hat{\lambda}_{5}\right)=-4(p-1)-2$. Hence we have $C_{\hat{\beta}_{4}}=C_{\hat{\beta}_{5}}+4(p-1)+2$. Applying formula $(*)$ to $\beta_{5}$ at the first crossing of the syllabus $a_{1}^{2}$, we get $C_{\hat{\beta}_{5}}=C_{\hat{\beta}_{6}}-l k\left(\hat{\lambda}_{6}\right)$ where $\beta_{6}=a_{1} a_{2}\left(a_{3} a_{1} a_{2}\right)^{m-2}$ and $\lambda_{6}=a_{1}^{2} a_{2}\left(a_{3} a_{1} a_{2}\right)^{m-2}$. We also have $l k\left(\hat{\lambda}_{6}\right)=-(p-1)-1=-p$. Hence we have $C_{\hat{\beta}_{5}}=C_{\hat{\beta}_{6}}+p$. In summary, we get

$$
C_{\hat{\beta}_{1}}=C_{\hat{\beta}_{6}}+9 p-2 .
$$

Now one can easily see that the claim follows.

Similarly we can treat the rest of cases. The proof of Proposition 2 is now complete.

\section{Proof of Proposition 3}

Given a 3 -braid $\beta$ in letters $a_{1}, a_{2}, a_{3}$, whose closure is a knot, there is a canonical way to construct a Seifert surface for $\hat{\beta}$ as follows: in the projection plane we have the braid diagram in its canonical form, place three rectangular disks in the space so that disk 1 lies in the projection plane and is on the left hand side of the braid, disk 3 also lies in the projection plane but on the right hand side of the braid, disk 2 lies perpendicularly above the projection plane, each disk having one side running parallel to the braid from top to the bottom, then to each letter $a_{1}\left(a_{1}^{-1}\right)$ occurring in $\beta$ use a half negatively (positively) twisted band connecting disks 1 and 2 , to each letter $a_{2}\left(a_{2}^{-1}\right)$ use a half negatively (positively) twisted band connecting disks 
2 and 3 , and to each letter $a_{3}\left(a_{3}^{-1}\right)$ use a half negatively (positively) twisted band connecting disks 1 and 3 (behind disk 2). Figure 6 illustrates such construction for $\beta=a_{1} a_{2} a_{3} a_{1}^{-1} a_{3}^{-1} a_{2}^{-1}$. We call the Seifert surface of $\hat{\beta}$ so constructed canonical Seifert surface of $\hat{\beta}$. In [18], it was proved that if $\beta$ is a 3 -braid in norm form (whose definition we recalled in Section 2), then its canonical Seifert surface has the minimal genus (thus is an essential and Thurston norm minimizing surface in the exterior of $\hat{\beta}$ ).

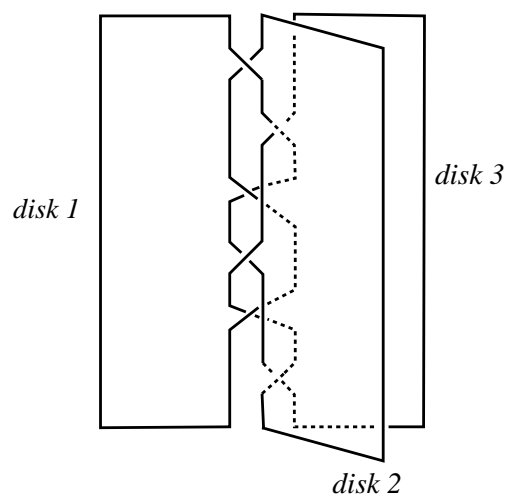

Fig. 6. Construction of the canonical Seifert surface.

Lemma 5. Let $\beta$ be a 3-braid such that $\hat{\beta}$ is a knot. Let $S$ be the canonical Seifert surface of $\beta$ and suppose that it has minimal genus.

(1) If $S$ contains two half twisted bands corresponding to the same letter $a_{1}$ or $a_{2}$ or $a_{3}$, then the -1-surgery on $\hat{\beta}$ is a manifold with essential lamination.

(2) If $S$ contains two half twisted bands corresponding to the same letter $a_{1}^{-1}$ or $a_{2}^{-1}$ or $a_{3}^{-1}$, then the 1-surgery on $\hat{\beta}$ is a manifold with essential lamination.

Proof. We shall only prove part (1) when $S$ contains two half twisted bands corresponding to the same letter $a_{1}$. All other cases can be proved similarly.

Let $M$ be the knot exterior of $\hat{\beta}$ in $S^{3}$. We shall also use $M(-1)$ to denote the manifold obtained by Dehn surgery on the knot $\hat{\beta}$ with the slope -1 . Let $V$ be the solid torus filled in $M$ to obtain the manifold $M(-1)$. We first construct an essential branched surface $B$ in the exterior $M$ and then prove that $B$ (which has boundary on $\partial M)$ can be capped off by a branched surface in $V$ to yield an essential branched surface $\hat{B}$ in $M(-1)$. The construction of $B$ is similar to that given in [14].

Since $S$ contains two half negatively twisted bands corresponding to $a_{1}$, there is a disk $D$ in $M$ as shown in Fig. 7(1) whose boundary lies in $S \cup \partial M$ and whose interior is disjoint from $S \cup \partial M$. With more detail, the boundary of $\partial D$ intersects $S$ in two disjoint arcs and intersects $\partial M$ in two disjoint arcs with the latter happening around 


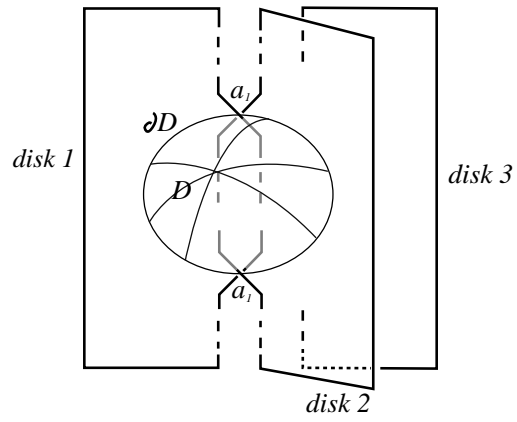

(1)

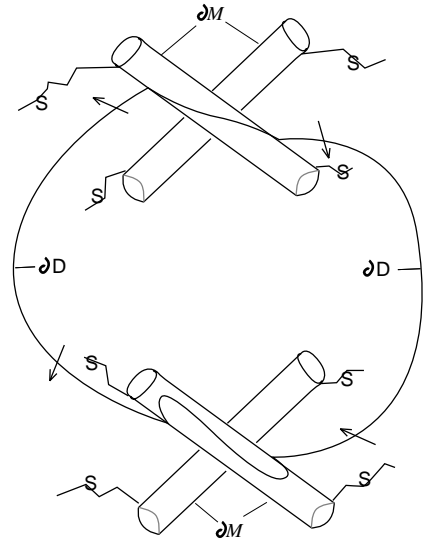

(2)

Fig. 7. Construction of $B$.

the places corresponding to the two bands of $a_{1}$. (Similar disks were used in [3] for a different purpose). The branched surface $B$ is the union of the Seifert surface $S$ and the disk $D$ with their intersection locus smoothed as shown in Fig. 7(2). The arrows in the figure indicate the cusp direction of branched locus. A similar argument as in [14] shows that the branched surface fully carries a lamination with no compact leaves and each negative slope can be realized as the boundary slope of a lamination fully carried by $B$. Since the disk $D$ intersects the knot exactly twice, the branched surface $B$ is essential in $M$ by [9, 3.12]. The branched locus of $B$ is a set of two disjoint arcs properly embedded in $S$, each being non-separating. The branched surface $B$ meets $\partial M$ yielding a train track in $\partial M$ as show in Fig. $8(1)$. Let $\mathcal{L}$ be a lamination fully carried by $B$ whose boundary slope is -1 . Then $\partial \mathcal{L}$ must look like that shown in Fig. 8(2).

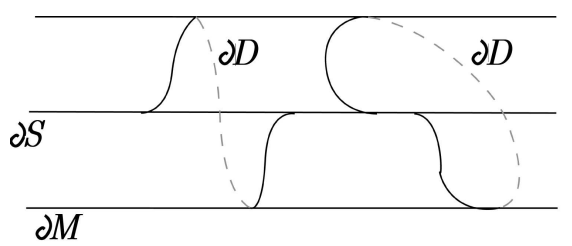

(1)

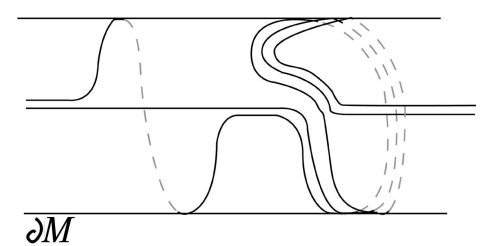

(2)

Fig. 8. (1) $\partial B$ on $\partial M,(2)$ the curve of slope -1 fully carried by $\partial B$.

We now construct a branched surface $B_{V}$ in the sewn solid torus $V$ such that the train track $B_{V} \cap \partial V$ is equivalent to the train track $B \cap \partial M$ and $B_{V}$ fully 
carries a lamination which is a set of meridian disks of $V$. Hence, $B$ and $B_{V}$ match together and form a branched surface $\hat{B}$ in $M(-1)$. Take a meridian disk $D_{0}$ of $V$ and push part of it near and around $\partial V$ as shown in Fig. 9(1) and then identify two disjoint sub-disks of $D_{0}$ as shown in Fig. 9(2). This gives a branched surface $B_{1}$ with the cusp direction along its singular locus (an arc) as shown in Fig. 9(2). Then we split $B_{1}$ locally at a place around a point of $\partial B_{1}$ as shown in Fig. $9(3)$ and then we start pinch the resulting branched surface along part of its boundary as shown in Fig. 9(4). The pinching continues as shown in Fig. 9(5) until we get the branch surface whose boundary is as shown in Fig. 9(6). The resulting surface is the branched surface $B_{v}$. Obviously the train track $\partial B_{v}$ on $\partial V$ is equivalent to the train track $\partial B$ in $\partial M$ and they can be matched in $\partial V=\partial M$.

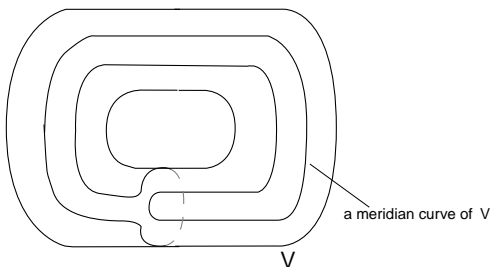

(1)

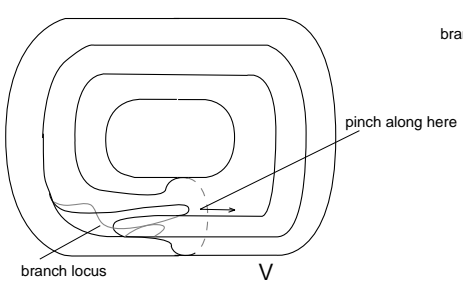

(4)

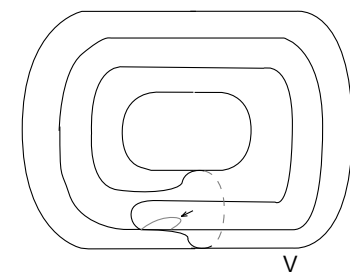

(2)

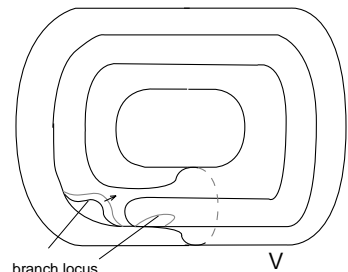

(3)

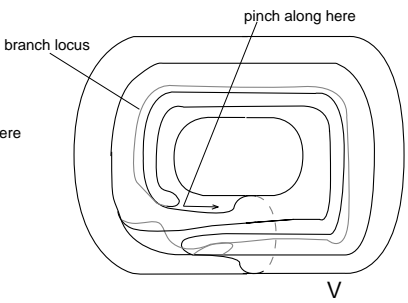

(5)

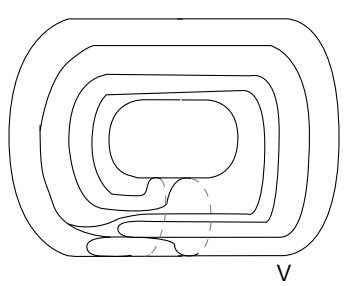

(6)

Fig. 9. Construction of $B V$.

To see that $\hat{B}$ is essential we have five things to check by [10, Definition 2.1]:

(i) $\hat{B}$ has no discs of contact;

(ii) the horizontal surface $\partial_{h} N(\hat{B})$ is incompressible and $\partial$-incompressible in $M(-1)-\stackrel{\circ}{N}(\hat{B})$, there are no monogons in $M(-1)-\stackrel{\circ}{N}(\hat{B})$ and no component of $\partial_{h} N(\hat{B})$ is a 2 -sphere;

(iii) $M(-1)-\stackrel{\circ}{N}(\hat{B})$ is irreducible;

(iv) $\hat{B}$ contains no Reeb branched surface;

(v) $\hat{B}$ fully carries a lamination. 
Condition (v) follows automatically from the construction since leaves of a lamination fully carried by $B$ with boundary slope -1 match on $\partial M=\partial V$ with (disk) leaves of a lamination fully carried by $B_{V}$. It also follows that $\hat{B}$ does not carry any compact surface since $B$ does not. Hence in particular condition (iv) holds also for $\hat{B}$. By the construction, one can easily see that $V-\stackrel{\circ}{N}\left(B_{v}\right)$ has two components, each of which topologically looks like as shown in Fig. 10. It follows that $M(-1)-\stackrel{\circ}{N}(\hat{B})$ is topologically the same as $M-\stackrel{\circ}{N}(B)$, with the same horizontal surface. From this we get conditions (ii) and (iii) for $\hat{B}$.

We now show that $\hat{B}$ has no disk of contact. Note that $\partial_{v}(N(\hat{B}))$ is a set of two annuli and each of the annuli is obtained from matching a component of $\partial_{v}(N(B))$ (a vertical disk) and a component of $\partial_{v}\left(N\left(B_{V}\right)\right)$ (a vertical disk). Hence, if $D_{c}$ were a contact disk in $N(\hat{B})$, then its boundary would have to intersect a component of $\partial_{v}(N(B))$. It follows that the interior of $D_{c}$ must enter into the region of $N(B)$ corresponding to a branch of $(S-$ the singular locus of $B)$. But one can easily see from Fig. 7 that the complement of the singular locus of $B$ in $S$ is a connected surface. It follows that $D_{c}$ has to intersect every $I$-fiber of $B$ since $D_{c}$ is transverse to $I$-fibers of $B$. In particular $\partial D_{c}$ has to intersect both of the vertical annuli of $\partial_{v}\left(\hat{B}_{v}\right)$, which gives a contradiction.

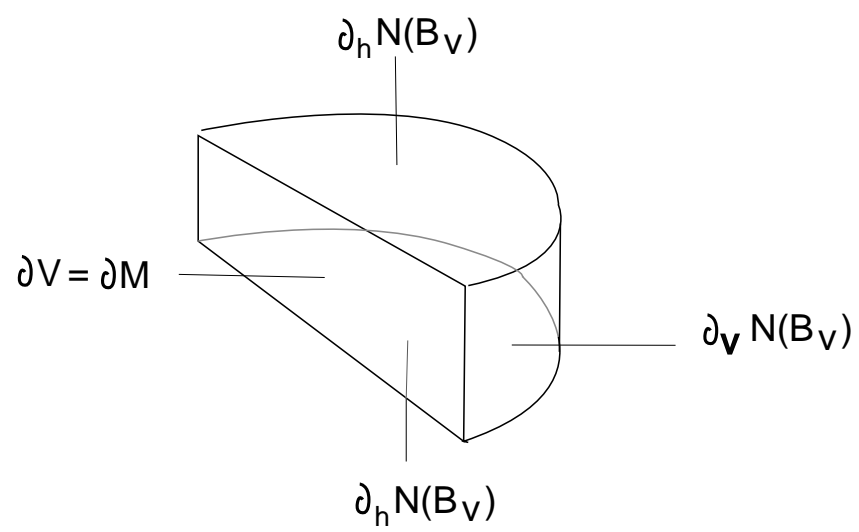

Fig. 10. A component of $V-\stackrel{\circ}{N}\left(B_{v}\right)$.

We now prove Proposition 3. By [18], the canonical Seifert surface of $\hat{\beta}$ has minimal genus. If the condition (1) of Proposition 3 holds, then the conclusion of Proposition 3 follows obviously from Lemma 5. Suppose that the condition (2) of Proposition 3 holds. To show that the 1-surgery on $\hat{\beta}$ gives a manifold with an essential lamination, we may assume, by Lemma 5 , that $\eta$ contains at most three syllabus, and they have different subscripts and all have power -1 . But $\eta$ contains at least two syllabuses. Suppose that the first and the second syllabuses 
of $\eta$ are $a_{3}^{-1}$ and $a_{2}^{-1}$, i.e. $\eta=a_{3}^{-1} a_{2}^{-1} \cdots$. Then since $\beta$ is a shortest word, the word $\delta$ does not end with a syllabus in $a_{3}$. Suppose that $\delta$ ends with a syllabus in $a_{1}$. Then we have $\beta=\cdots a_{1} a_{3}^{-1} a_{2}^{-1} \cdots$. By a band move isotopy of the Seifert surface as shown in Fig. 11(1) we get an isotopic 3-braid $\beta^{\prime}$ which contains two $a_{2}^{-1}$. (Algebraically, $\beta=\cdots a_{1} a_{3}^{-1} a_{2}^{-1} \cdots=\cdots a_{2}^{-1} a_{1} a_{2}^{-1} \cdots=\beta^{\prime}$ ). Further the canonical Seifert surface of $\hat{\beta}^{\prime}$ is isotopic to that of $\hat{\beta}$ and thus has minimal genus. So we may apply Lemma 5 to see that for the knot $\hat{\beta}^{\prime}=\hat{\beta}$, the 1 -surgery gives a manifold with essential lamination. Suppose then that $\delta$ ends with a syllabus in $a_{2}$. Since $\delta$ is assumed to contain at least two syllabuses, $\beta=\cdots a_{1} a_{2}^{k} a_{3}^{-1} a_{2}^{-1} \cdots$. Again we may first use the band-isotopy as shown in Fig. 11(2) and then use the band isotopy of Fig. 11(1) to get an isotopic 3-braid whose canonical Seifert surface contains two bands corresponding to $a_{2}^{-1}$. Hence Proposition 3 follows from Lemma 5 in this case as well. Similarly one can treat the cases when $\eta$ starts with $a_{2}^{-1} a_{1}^{-1}$ or with $a_{1}^{-1} a_{3}^{-1}$. The case when $\delta$ contains at most three syllabuses, each having power at most one, can be proved similarly. This proves Proposition 3 under its condition (2). Finally if the condition (3) of Proposition 3 holds then either condition (2) of Proposition 3 holds or one can get directly two letters $a_{i}$ of the same subscript in $\delta$ and two letters $a_{j}^{-1}$ of the same subscript in $\eta$. So again the Proposition follows from Lemma 5 .
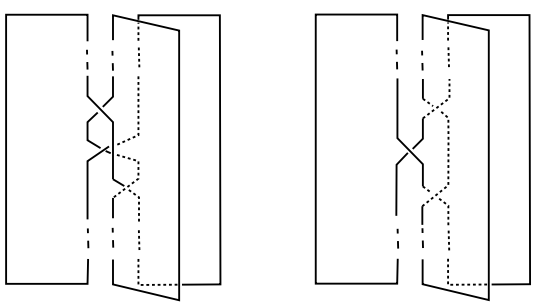

(1)

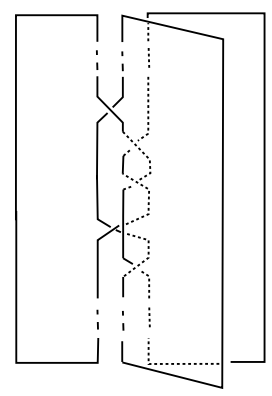

(2)

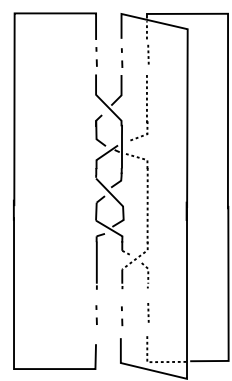

)

Fig. 11. The band move isotopies.

\section{Acknowledgements}

We would like to thank a previous referee whose suggestions simplify our original proof of Proposition 2. 


\section{References}

[1] S. Akbulut and J. McCarthy, Casson's invariant for oriented homology 3-spheres, Princeton University Press (1990).

[2] R. Bing and J. Martin, Cubes with knotted holes, Trans. Amer. Math. Soc. 155 (1971). 217-231.

[3] J. Birman and W. Menasco, Studying links via closed braids. III. Classifying links which are closed 3-braids, Pacific J. Math. 161 (1993) 25-113.

[4] S. Bleiler and M. Scharlemann, A projective plane in $R^{4}$ with three critical points is standard. Strongly invertible knots have property $P$, Topology 27 (1988) 519-540.

[5] M. Culler, C. M. Gordon, J. Luecke and P. Shalen, Dehn surgery on knots, Ann. Math. 125 (1987) 237-300.

[6] C. Delman and R. Roberts, Alternating knots satisfy strong property P, Comment. Math. Helv. 74 (1999) 376-397.

[7] N. Dunfield, Cyclic surgery, degrees of maps of character curves, and volume rigidity for hyperbolic manifolds, Invent. Math. 136 (1999) 623-657.

[8] D. Gabai, Surgery on knots in solid tori, Topology 28 (1989) 1-6.

[9] —, Foliations and the topology of 3-manifolds, J. Differential Geom. 18 (1983) 445503.

[10] D. Gabai and U. Oertel, Essential laminations and 3-manifolds, Ann. Math. 130 (1989) 41-73.

[11] C. Gordon and J. Luecke, Knots are determined by their complements, J. Amer. Math. Soc. 2 (1989), no. 2, 371-415.

[12] J. Hempel, A simply connected 3-manifold is $S^{3}$ if it is the sum of a solid torus and the complement of a torus knot, Proc. Amer. Math. Soc. 15 (1964) 154-158.

[13] R. Kirby, Problems in Low-Dimensional Topology, in Geometric Topology, Ed. William H. Kazez, AMS/IP Studies in Advanced Mathematics, 2.2. (1997) 35-473.

[14] R. Roberts, Constructing taut foliations, Comment. Math. Helv. 70 (1995) 516-545.

[15] D. Rolfsen, Knots and Links, Publish or Perish 1976.

[16] J. Van Buskirk, Positive knots have positive Conway polynomial, Lecture Notes in Math. 1144 (1985) 146-159.

[17] Y. Wu, Dehn surgery on arborescent knots, J. Differential Geom. 43 (1996) 171-197.

[18] P. Xu, The genus of closed 3-braids, J. Knot Theory Ramifications 1 (1992) 303-326. 